\title{
PROCESOS DE AMERICANIZACIÓN LÉXICA EN LA HISTORIA DEL ESPAÑOL SALVADOREÑO: LA CARTA-RELACIÓN DE DIEGO GARCÍA DE PALACIO COMO EJEMPLO*
}

\author{
José Luis Ramírez Luengo y José Armando San Martín Gómez \\ Universidad Autónoma de Querétaro, México
}

\section{RESUMEN}

Quizá uno de los aspectos más relevantes de la historia del español sea la americanización que experimenta a su llegada al Nuevo Mundo, por medio de la cual se transforma en un instrumento útil para la expresión de la realidad del continente; esta adaptación se produce, en el caso del léxico, a través de una serie de procesos que implican diversas estrategias como la incorporación de voces autóctonas o la modificación del fondo endohispánico, las cuales tienen lugar desde muy pronto y afectan tanto a los criollos como a los peninsulares que emigran desde Europa. Partiendo, pues, de esta situación, el trabajo se propone describir los procesos mencionados que aparecen en la carta que Diego García de Palacio escribe desde los actuales territorios salvadoreños a fines del siglo XVI, con el propósito de constatar no solo la presencia de las estrategias indicadas, sino también las preferencias que se descubren al respecto en este escrito.

Palabras clave: historia del español de América, léxico, americanización, El Salvador, siglo XVI.

\author{
PROCESSES OF LEXICAL AMERICANIZATION IN THE HISTORY \\ OF SALVADORIAN SPANISH: DIEGO DE PALACIO'S CARTA-RELACIÓN AS EXAMPLE
}

\section{Abstract}

One of the most relevant aspects of the history of Latin American Spanish may be the americanization that this language undergoes when it arrives to America, in order to transform it in a useful tool to express American reality. In the case of lexicon, this adaptation takes place through two different strategies: the incorporation of loanwords from American languages or the semantic modification of Spanish lexical items. Both processes happen very soon and they are found not only in creoles' writings, but also in Peninsular emigrants' ones. This paper aims to describe these processes as they appear in an official document that Diego Garcia de Palacio writes at the end of 16th. century from nowadays Salvadorian region. Its main goal is not only to verify the use of both strategies in this corpus, but also to point out the usage preferences that the author shows in his text.

Keywords: History of Latin American Spanish, lexicon, americanization, El Salvador, 16th century. 
No cabe duda de que uno de los aspectos más relevantes en la historia del español lo constituye la americanización que experimenta esta lengua a su llegada al Nuevo Mundo, a través de la cual un medio de comunicación preparado para ser empleado en Europa se transforma en un instrumento útil para la expresión de la realidad del continente (Ramírez Luengo, 2017: 605); esta adaptación, que se produce en todos los niveles lingüísticos, es sin duda especialmente evidente en el caso del vocabulario, en el que tales procesos, dialectalmente diferenciados, van a terminar por crear «un mapa léxico propio que va a identificar a una región por medio de un conjunto de voces que, sean conocidas solamente en la zona o tengan un significado especial en ese lugar, constituyen un rasgo de identidad que distingue esa variedad del español de todas las demás del mundo hispánico» (Ramírez Luengo, 2012: 395).

Por lo que se refiere a la manera como se produce tal americanización, para el caso concreto del léxico se ha apuntado ya una serie de procesos que se pueden englobar en dos grandes mecanismos: la acogida de voces tomadas de las lenguas autóctonas y la alteración significativa de diversas unidades del fondo endohispánico (Buesa Oliver y Enguita Utrilla, 1992), es decir, lo que se ha dado en llamar estrategia de incorporación y estrategia de modificación respectivamente (Ramírez Luengo, 2017: 605); dichos mecanismos se caracterizan no solo por hacerse presentes desde antiguo en todas las hablas que conforman el español de América -si bien en porcentajes diferentes, lo que produce distintas configuraciones léxicas regionales ${ }^{1}-$, sino también por afectar tanto a los criollos como a los indígenas que adquieren el español, e incluso los peninsulares que emigran desde Europa y se incorporan a las distintas sociedades coloniales (Frago, 1999: 227-243; Ramírez Luengo, 2013, 2014).

Partiendo, pues, de la situación que se acaba de describir, este trabajo se propone analizar los procesos de americanización léxica presentes en la carta-relación que a finales del siglo Xvi escribe el oidor García de Palacio acerca de los actuales

* Este trabajo constituye uno de los productos resultantes del proyecto de investigación Léxico histórico del español de El Salvador tardocolonial (1650-1803) (FFL-2018-02), financiado por la Universidad Autónoma de Querétaro por medio del Fondo para el Fortalecimiento de la Investigación UAQ-2018.

1 Este concepto se define como «la preferencia que muestra una determinada variedad dialectal por una de las posibles estrategias (modificación/incorporación) que se emplean a la hora de dialectalizar el léxico» (Ramírez Luengo, 2017: 605-606), y está fuertemente influenciado por factores de tipo sociohistórico; para un primer análisis de tales factores, así como para la ejemplificación concreta de este concepto en una variedad americana específica -el oriente boliviano del siglo XVIII-, véase Ramírez Luengo (2017). 
territorios salvadoreños (Escalante Arce, 2000), con la intención no solo de constatar la presencia de las estrategias indicadas más arriba, sino también de señalar las diferencias porcentuales que se descubren al respecto en este escrito, y poder así detectar los orígenes históricos de los resultados que, desde este punto de vista, muestra actualmente el español centroamericano en general y salvadoreño en particular. En este sentido, los objetivos específicos que se persiguen son los siguientes: a) señalar las marcas textuales que ponen de manifiesto la americanización léxica; b) analizar las estrategias de modificación e incorporación que se descubren en la obra; c) establecer la preferencia que muestra García de Palacio por alguna de las estrategias mencionadas; $\mathrm{y} d$ ) detectar posibles divergencias en su empleo de acuerdo con el campo léxico considerado.

Por lo que se refiere al autor, conviene señalar que Diego García de Palacio, nacido en el norte peninsular en 1542, se traslada al Reino de Guatemala como oidor de su Real Audiencia en 1573, y allí desarrolla importantes tareas administrativas hasta finales de esta década (Escalante Arce, 2019); tras pasar a México, sigue su carrera burocrática en la corte virreinal y llega incluso a ser rector de su universidad durante un ańo, si bien posteriormente es acusado de «fraudes y mala administración en las diligencias que se le encargaron como oidor de la Real Audiencia de México» y termina por morir en la capital novohispana en 1595 (Escalante Arce, 2000: 12) ${ }^{2}$.

En cuanto a la obra en sí -conservada actualmente en la biblioteca de la Real Academia de la Historia, en $\mathrm{Madrid}^{3}$-, cabe mencionar que no es el único escrito del autor, ni probablemente el más importante ${ }^{4}$ : en concreto, se trata de una relación relativamente breve dirigida al rey, que se fecha en Santiago de los Caballeros el 8 de marzo de 1576 y en la que se describen muy cuidadosamente los territorios de las alcaldías mayores de Sonsonate y San Salvador, así como la naturaleza de la región y las costumbres de los naturales; constituye -en palabras de Escalante Arce (2000: 11) - «la primera descripción geográfica, de elementos culturales y del entorno natural de la parte del territorio que visitó, con información de primera mano sobre los nahua-pipiles», y es precisamente por esta cercanía personal a la realidad que relata por lo que resulta de primera importancia para realizar un análisis como el presente, enfocado en la exposición de los procesos de modificación léxica

${ }^{2}$ La peripecia vital de García de Palacio ha sido estudiada por varios investigadores; a este respecto, véanse Sainz López-Negrete (1978), Arróniz (1994) y Cuesta Domingo (1994).

${ }^{3}$ Dadas las dificultades existentes para acceder al original, en este caso se ha trabajado con la edición preparada por Escalante Arce (2000) para la editorial Concultura, cuyas lecturas se han seguido en todas las ocasiones.

${ }^{4}$ En efecto, su formación militar y náutica lo lleva a escribir tratados relacionados con estas temáticas, en concreto sus Diálogos militares en la formación e información de personas e instrumentos y cosas necesarias para el buen uso de la guerra (Ciudad de México: Pedro de Ocharte, 1587) y una Instrucción náutica para navegar (Ciudad de México: Pedro de Ocharte, 1583) (Escalante Arce, 2019); esta última obra es la que más se ha analizado desde el punto de vista lexicológico, con estudios como Kero (2002), García-Macho (2004) o -junto con otros tratadistas-Carriazo Ruiz (2002). 
-americanización, si se quiere- que experimenta el habla de un peninsular a la hora de expresar las novedosas entidades a las que se enfrenta en su nuevo entorno vital.

Como se ha señalado en múltiples ocasiones, a su llegada a América los españoles descubren rápidamente la incapacidad que presenta su idioma para nombrar los referentes desconocidos que les ofrece el continente (Ramírez Luengo, 2007: 71), de manera que se ven obligados a «adaptar su herramienta de comunicación y hacerla útil para esa nueva realidad a la que se enfrentan, esto es, americanizar la lengua para poder explicar, así, el nuevo mundo que los rodea» (Ramírez Luengo, 2017: 605). Por supuesto, este proceso resulta prácticamente inmediato, de manera que no sorprende que García de Palacio evidencie ya en el texto analizado la existencia de tales fenómenos de americanización, en su caso por medio del empleo de fórmulas como que acá llaman y otras semejantes, tanto aplicadas a indigenismos como a voces endohispánicas que han sufrido una modificación semántica (ejemplos 1 y 2):

1) Tiene toda esta costa muchas praderías, que acá llaman sabanas, grandes y de mucho pasto (p. 37).

2) Es por el verano, que acá llaman desde noviembre hasta mayo (p. 44).

Teniendo en cuenta, por tanto, la aparición en el corpus de estudio de los procesos que interesa estudiar, se hace ahora necesario analizar las estrategias por medio de las cuales se describe la realidad americana, que -como se dijo más arribavan a ser fundamentalmente dos, la modificación y la incorporación.

Por lo que se refiere a la primera de ellas, la modificación supone "partir del léxico existente para modificarlo -formal o semánticamente- y adaptarlo así a la nueva realidad» (Ramírez Luengo, 2017: 605), proceso que conlleva la resemantización de la voz en cuestión por medio del establecimiento de una comparación entre el referente conocido y la nueva realidad americana; como era de esperar, esta estrategia no está ausente de la relación del oidor de Guatemala, y de hecho se registran 16 elementos que, de acuerdo con las informaciones del DLE (2014) y el DAMER (2010), cumplen la definición que se acaba de facilitar, a saber: almácigo, anime, arfar, bálsamo, ciruelo, encomendar, encomendero, estancia, indio, invierno, mazorca (de cacao), mojarra, nata, piña, venado, verano ${ }^{5}$. Cabe indicar que, si bien en cier-

5 Junto a estos americanismos semánticos (Company, 2010: XVII), se registra también otra serie de elementos endohispánicos que presentan una modificación significativa por comparación, si bien puramente ocasional: esto es lo que ocurre, por ejemplo, cuando se asocian las autoridades religiosas indígenas a los cargos de la Iglesia católica, con casos como papa ("Allende del cacique y señor natural, tenían un papa que llamaban tecti»; p. 47) o mayordomo («Salía el papa de su casa con el sabio mayordomo, y subían al cu [k'u] con el cacique y principales»; p. 48). Aunque en principio parecen cumplir la definición que se ha aportado anteriormente y por tanto podrían incorporarse 
tas ocasiones el autor del texto señala de forma explícita -y con distintas estrategias discursivas- la diferencia semántica existente entre el uso peninsular y americano del mismo término (ejemplos 3 y 4), en otras muchas las voces se emplean con el significado nuevo, propio del Nuevo Mundo, sin ningún comentario acerca de su valor semántico, lo que parece demostrar bien a las claras la generalización de este en la variedad centroamericana del español y, como consecuencia de lo anterior, en el idiolecto del propio autor del texto ${ }^{6}$ (ejemplos 5 y 6 ).

3) Hay en toda esta tierra un árbol común, que nosotros llamamos ciruelo (p. 38).

4) Tuve noticias que había en él venados de la forma que son los que en la Yndia [India] (p. 50).

5) Y entre ellos conté, tasé y repartí setenta y ocho de lo que los naturales deben pagar de tributo a cada año a sus encomenderos (p. 36).

6) $\mathrm{Al}$ presente hay algunas estancias, pero muy poco ganado para lo que podría haber (p. 46).

Ahora bien, no es esta la única estrategia presente en el corpus para producir la americanización del vocabulario: en efecto, junto a ella se descubren también ejemplos de la estrategia de incorporación, que implica «la introducción de unidades léxicas nuevas [...] tomadas de diferentes sistemas lingüísticos, generalmente los autóctonos del Nuevo Mundo» (Ramírez Luengo, 2017: 605), y que en principio el autor emplea cuando la novedad del referente le impide establecer los procesos de

como casos de la estrategia de modificación, el hecho de que se trate de un uso puramente ocasional que no se consolida obliga a considerarlos de manera diferenciada, como simples comparaciones entre referentes; resulta de interés seńalarlos, con todo, porque parecen mostrar el primer paso del proceso que va a llevar a la creación de los americanismos semánticos, y porque obligan a preguntarse, además, de qué manera objetiva es posible distinguir entre estos elementos y los americanismos semánticos propiamente dichos, cuestión teórica de gran interés que será necesario analizar con cuidado en posteriores trabajos. Asimismo, el corpus ofrece algunos casos de palo con valor de 'árbol' («el otro palo si lo echan en el agua se torna azul extremadamente»; p. 44) cuyo empleo actual, restringido a varios países de América ( $D L E, 2014$ : s.v. palo), transforma hoy en día a este elemento en americanismo semántico, si bien no lo era en la época que se está analizando, tal y como demuestran los datos de CORDE (2019), donde esta significación aparece de manera frecuente en la España del Quinientos; esto demuestra, una vez más, el carácter dinámico del concepto americanismo, cuestión que ha sido ya planteada teóricamente (Ramírez Luengo, 2012) y analizada en diferentes trabajos (Ramírez Luengo, 2017, 2019), pero cuya trascendencia para la historia del léxico americano conviene volver a recordar. Por último, es también importante señalar la aparición en ocasiones de voces referidas a la flora y la fauna -a manera de ejemplo, naranjo: «Tiene sus hojas como castañal, aunque medianas, produce flor y fruta casi todas las lunas y lo mismo hacen en estas partes todos los naranjos» (p. 40)- que plantean notables dificultades de interpretación, habida cuenta de la dificultad de establecer a partir de los textos si el autor lo está aplicando a la realidad peninsular (voz común) o a una propiamente americana (americanismo semántico).

${ }^{6}$ Se trata, pues, de un claro ejemplo de las consecuencias léxicas que tienen las situaciones de contacto interdialectal, cuestión que no interesa para los objetivos de este trabajo, pero que ha sido analizada con cierto detalle en el caso de otros peninsulares emigrados a América (Ramírez Luengo, 2013, 2014). 
comparación/metaforización que exige la modificación descrita en el párrafo anterior $^{7}$; por lo que se refiere a estos elementos, son 29 los indigenismos -tanto integrados (ejemplos 7-8) como no integrados (ejemplos 9-10) ${ }^{8}$ - que se incorporan al texto, tal y como se recoge en la lista siguiente: ají, axín, cacao, cacique, caimán, calpul, chalchihuite, chián, copal, cote, cu, elote, hule, jicara, jicol, maiz, maxtli, milpa, mitote, nahuite, quetzal, sabana, tecuhtli, tehuamatini, teupa, teupixqui, xequipil, zapote y zontle.

7) Tiene las calidades del suelo y cielo que la de Guazacapán, y abundancia de cacao, pesca y frutas y demás cosas que acá comúnmente hay en las tierras calientes (p. 40).

8) Los que eran soldados de la guerra no dormían en sus casas con sus mujeres, sino en unos calpules que tenían diputados para ello (p. 49).

9) $Y$ los sacerdotes tomaban la sangre del sacrificado en cuatro jicaras, que son unos vasos de cierta fruta que los indios usan (p. 49).

10) Un manojo de plumas muy buenas de unos pájaros que hay en esta tierra que llaman quetzales (p. 47).

Una vez comprobada, por tanto, la presencia de las dos grandes estrategias de americanización léxica en el corpus de estudio, parece posible extraer dos conclusiones de relativo interés para la mejor comprensión histórica de este fenómeno: en primer lugar $-\mathrm{y}$ como era de esperar-, que tales procesos de adaptación a la nueva realidad que experimenta el vocabulario del español centroamericano se producen

7 Con todo, es más que probable que este no sea el único factor que favorece la incorporación de voces autóctonas, y que haya otras cuestiones - por ejemplo, la intención de añadir más precisión al texto, o la finalidad que este persigue, especialmente evidente en tratados que procuran describir la naturaleza o la sociedad americanas- que también influyan en el empleo de esta estrategia de americanización.

${ }^{8}$ Se entienden como indigenismos integrados aquellos que se puede suponer se han incorporado ya al habla de la región, mientras que los no integrados coinciden con los ocasionalismos, esto es, "palabras que no pertenecen al uso habitual de la lengua receptora, sino que se usan ocasionalmente en ella [...] con plena conciencia de su condición de extranjeras y sin voluntad de integrarlas» (Álvarez de Miranda, 2009: 144); en principio, se suele establecer la diferencia entre ambos grupos a partir de la existencia o no de una glosa que explique los elementos en cuestión, si bien este criterio no deja de ser discutible, dado que el hecho de que aparezca tal explicación en este texto específico quizá no se pueda interpretar como «el grado de integración de esas voces en el español centroamericano", sino más bien el que presentan "en el idiolecto de un hablante concreto" (Ramírez Luengo, en prensa), en este caso el peninsular García de Palacio. En concreto, y de acuerdo con este criterio, los vocablos integrados son 16 (ají, cacao, cacique, caimán, calpul, chián, copal, cu, elote, jicol, maiz, maxtli, milpa, teupa, hule, mitote), mientras que los no integrados se reducen a trece (axin, chalchihuite, cote, jicara, nahuite, quetzal, sabana, tecubtli, tehuamatini, teupixqui, xequipil, zapote, zontle). A esto se tienen que sumar otros indigenismos que, por pertenecer al campo de la onomástica -tanto teónimos (Itzqueye, Quetzalcóatl) como glotónimos (popoluca, potón, cholulteca) y nombres de pueblos autóctonos (pipiles, chontales)-, no se han considerado en este listado.

9 Para la constatación de su origen etimológico se ha empleado fundamentalmente la información registrada en Santamaría (1959), el DCECH (1980-1991) y Morínigo (1998). 
de una manera muy rápida, habida cuenta de su aparición en los textos que se redactan en momentos tan tempranos como la segunda mitad del siglo XVI; en segundo lugar, que si bien es verdad que ambas estrategias hacen acto de presencia en el corpus, también lo es que García de Palacio parece mostrar una especial predilección por la incorporación, tal y como evidencian los datos siguientes (tabla 1):

\begin{tabular}{lcl}
\hline & \multicolumn{2}{c}{$\begin{array}{l}\text { TABLA 1. ESTRATEGIAS DE AMERICANIZACIÓN LÉXICA } \\
\text { EN EL CORPUS (MODIFICACIÓN VS. INCORPORACIÓN) }\end{array}$} \\
\hline EstratEgIa & CASOS & \multicolumn{1}{c}{ VOCES } \\
\hline Modificación & $16(35.55 \%)$ & $\begin{array}{l}\text { almácigo, anime, arfar, bálsamo, ciruelo, encomendar, encomendero, estancia, } \\
\text { indio, invierno, mazorca (de cacao), mojarra, nata, piña, venado, verano }\end{array}$ \\
\hline Incorpación & $29(64.44 \%)$ & $\begin{array}{l}\text { ají, axin, cacao, cacique, caimán, calpul, chalchihuite, chián, copal, cote, cu, } \\
\text { elote, hule, jicara, jicol, maiz, maxtli, milpa, mitote, nahuite, quetzal, sabana, } \\
\text { tecuhtli, tehuamatini, teupa, teupixqui, xequipil, zapote, zontle }\end{array}$ \\
\hline Total & $45(100 \%)$ & \\
\hline
\end{tabular}

De este modo, los datos anteriores permiten en principio constatar que, en la configuración léxica que refleja el corpus analizado -entendido este concepto, según se dijo ya, como «la preferencia que muestra una determinada variedad dialectal por una de las posibles estrategias (modificación/incorporación) que se emplean a la hora de dialectalizar el léxico» (Ramírez Luengo, 2017: 605-606)-, parece haber una clara preferencia por la incorporación, que representa prácticamente el $65 \%$ de los vocablos que evidencian la adaptación léxica del español a la realidad americana; con todo -y sin negar esta primera conclusión-, es probable que un acercamiento más detallado, en el que se tengan en cuenta los distintos campos semánticos a los que pertenecen los distintos referentes, permita obtener una visión más profunda de la cuestión (tabla 2$)^{10}$.

\begin{tabular}{lll}
\hline & $\begin{array}{c}\text { TABLA 2. ESTRATEGIAS DE AMERICANIZACIÓN LÉXICA } \\
\text { EN EL CORPUS (POR CAMPO LÉXICO) }\end{array}$ \\
\hline \multicolumn{1}{c}{ CAMPO LÉXICO } & \multicolumn{1}{c}{ MODIFICACIÓN } & \multicolumn{1}{c}{ INTEGRACIÓN } \\
\hline Instituciones sociales & encomendar, encomendero, indio & $\begin{array}{l}\text { cacique, mitote, nahuite, tecubtli, tehuamatini, } \\
\text { teupixqui, xequipil, zontle }\end{array}$ \\
\hline Industria/construcción & nata & axín, calpul, cu, teupa \\
\hline Alimentación & aji \\
\hline
\end{tabular}

10 Dadas las múltiples clasificaciones por campo léxico existentes (Mejías, 1980, Polo Cano, 2005) y las notables diferencias que se registran entre ellas, en este caso se ha optado por partir de la establecida en Ramírez Luengo (2019: 21) y modificarla de acuerdo con los propios datos del corpus; esto no hace desaparecer, por supuesto, la notable carga de subjetividad que cualquiera de estas taxonomías presenta. 


\begin{tabular}{|c|c|c|}
\hline Flora/agricultura & $\begin{array}{l}\text { almácigo, anime, bálsamo, ciruelo, } \\
\text { mazorca (de cacao), piña }\end{array}$ & $\begin{array}{l}\text { cacao, chian, copal, cote, elote, hule, jicara, maiz, } \\
\text { milpa, zapote }\end{array}$ \\
\hline Enseres/utensilios & & chalchihuite, jicol, maxtli \\
\hline Clima/geografía & invierno, verano & sabana \\
\hline Fauna/ganadería & estancia, mojarra, venado & caimán, quetzal \\
\hline Otros & arfar & \\
\hline TOTAL & $16(35.55 \%)$ & $29(64.44 \%)$ \\
\hline
\end{tabular}

En efecto, a pesar de su relativa escasez -y de que, por tanto, estas primeras conclusiones en modo alguno resultan concluyentes-, los datos del corpus parecen evidenciar unas primeras diferencias de cierta relevancia en lo que se refiere a la estrategia de americanización que privilegian los distintos campos léxicos: así, mientras que en el caso de las instituciones sociales o la flora/agricultura la integración es claramente mayoritaria -con porcentajes del $61.53 \%$ y del $62.5 \%$ de esta estrategia respectivamente-, en el caso de categorías como la fauna/ganadería o el clima/ geografía la preferencia se invierte, y en estos casos es la modificación la estrategia predominante, con valores de entre el $60 \%$ y $66.6 \%$ del total. Es posible sostener, por tanto, que, más allá de la configuración léxica general de una variedad dialectal concreta, se hace preciso atender también a cómo se desarrollan las estrategias de americanización en campos nocionales específicos, que no se comportan necesariamente de la misma manera y cuyas diferencias responden a circunstancias sociohistóricas de naturaleza muy variada -tales como el mayor o menor peso de la influencia indígena en esa realidad específica, o el grado de novedad y distancia de esta respecto a lo que supone la experiencia previa de los españoles ${ }^{11}$ - que es necesario tener en cuenta para lograr una mejor comprensión de las razones últimas que explican los procesos que se están describiendo en estas páginas ${ }^{12}$.

${ }^{11}$ De hecho, no es casualidad que en el corpus la integración se descubra de manera predominante en realidades como los enseres y utensilios, las instituciones sociales y la flora/agricultura, es decir, en campos nocionales que responden perfectamente a los factores que se acaban de señalar.

${ }_{12}$ Del mismo modo, y desde un punto de vista metodológico, hay que tener en cuenta también que «la tipología textual que constituye el corpus de análisis puede distorsionar la -aparenteconfiguración léxica de una variedad concreta del español» (Ramírez Luengo, 2017: 608), dada la tendencia más o menos marcada, por ejemplo, a incorporar indigenismos según la finalidad del texto en cuestión o la identidad del receptor a quien va dirigido; teniendo esto en cuenta, resulta del todo necesario responder a una serie de interrogantes acerca de cómo minimizar esta cuestión -tales como qué características tiene que cumplir un corpus para evitar este problema, si existe alguna tipología textual concreta que no produzca estas distorsiones o cuál es el tamaño suficiente del corpus para poder paliarlas (Ramírez Luengo, 2017: 608)-, o al menos reflexionar sobre cómo incorporar este hecho al análisis para obtener una visión lo más realista posible de la configuración léxica de una variedad lingüística en un momento específico de su historia. 
Por otro lado, y más allá de los procesos descritos hasta el momento, la aparición en el corpus de lo que se ha dado en llamar americanismos de frecuencia ${ }^{13}$ pone de manifiesto cómo en ocasiones la dialectalización léxica del español del Nuevo Mundo puede adquirir una forma más sutil que las que se han descrito hasta el momento, la cual se refleja generalmente en la imposición y/o generalización diatópica de un vocablo concreto frente a otras posibilidades presentes también en el sistema; en concreto, los datos del corpus parecen demostrar que se trata del proceso cuantitativamente más escaso, habida cuenta de que se registra apenas en los siete elementos que, junto a sus frecuencias de uso espańola y americana, se presentan en la tabla siguiente (tabla 3$)^{14}$ :

\begin{tabular}{lcc}
\hline & $\begin{array}{c}\text { TABLA 3. ESTRATEGIAS DE AMERICANIZACIÓN LÉXICA } \\
\text { EN EL CORPUS (AMERICANISMOS FRECUENCIA) }\end{array}$ \\
\hline \multicolumn{1}{c}{ Voces } & España (casos/frecuencia) & AmÉrica (casos/frecuencia) \\
\hline Danta & $0(0.0)$ & $51(13.14)$ \\
\hline Desaguadero & $10(0.21)$ & $60(15.46)$ \\
\hline Durazno & $152(3.30)$ & $28(7.21)$ \\
\hline Estero & $6(0.13)$ & $211(54.39)$ \\
\hline Peńol & $22(0.47)$ & $364(93.83)$ \\
\hline Perulero & $11(0.23)$ & $13(3.35)$ \\
\hline Poblazón & $5(0.10)$ & $487(125.53)$ \\
\hline
\end{tabular}

Como se puede ver en los datos anteriores $-\mathrm{y}$ dejando de lado algunos casos que pueden resultar hasta cierto punto problemáticos ${ }^{15}-$, en todos los elemen-

13 Tales americanismos se definen, en palabras de Company (2010: XVII), como «voces o construcciones compartidos, en forma y significado, con el español peninsular castellano, pero que muestran en América una mucho mayor frecuencia de empleo y de generalización». Cabe indicar que se trata del subtipo más difícil de delimitar históricamente, no solo por las complicaciones que supone establecer la frecuencia de uso de un vocablo en el pasado, sino también por los múltiples problemas que plantea desde un punto de vista teórico, algunos de los cuales se presentan ya en Ramírez Luengo (2017: 614-615).

14 Como en trabajos anteriores (Ramírez Luengo, 2017, 2019), los americanismos de frecuencia se han establecido por medio de la comparación de los casos por millón de palabras (CMP) de estas voces que, con todas las grafías posibles, ofrece CORDE (2019) en España y América en el periodo cronológico 1501-1600; teniendo en cuenta tales resultados -y extrayendo del cálculo los ejemplos presentes en la onomástica (en concreto, Desaguadero y Santiago del Estero)-, se han incorporado a la lista de este subtipo de americanismos aquellos elementos cuya frecuencia de uso en textos americanos (o españoles de temática americana) dobla al menos la que se registra en el subcorpus español.

${ }_{15}$ En concreto, en los casos de perulero y durazno la escasa distancia existente entre las frecuencias española y americana plantea la duda de si tales elementos pertenecen ya a este subtipo de americanismos en el momento estudiado o si las diferencias registradas tienen que ver con cuestiones que van más allá del uso, como, por ejemplo, los textos que componen el corpus; en el caso de danta, la no aparición de ejemplos españoles en el corpus obliga a interpretarlo más bien como un caso de 
tos mencionados es posible detectar una diferencia entre la frecuencia de uso espańola y americana que resulta muy marcada y que se encuentra notablemente por encima de la que teóricamente se ha fijado para el establecimiento de estos elementos; en este sentido, son especialmente elocuentes en el corpus los casos de peñol y estero, con frecuencias de empleo 200 y 400 veces superiores en América, así como muy especialmente poblazón, cuyos usos americanos superan 1255 veces las apariciones por millón de palabras del corpus de España. Se puede concluir a la luz de estos datos, por tanto, que ya desde la segunda mitad del siglo XVI es posible descubrir "ciertas preferencias léxicas que, dentro del vocabulario español, manifiestan las diversas variedades del Nuevo Mundo, en un claro proceso de selección normativa que también contribuye -y no poco- a la dialectalización de este nivel lingüístico» (Ramírez Luengo, 2017: 69), lo que sin duda resulta de gran interés no solo para comprender de manera más profunda cómo las distintas variedades americanas van adoptando una personalidad léxica diferenciada, sino también para constatar la celeridad con que tal fenómeno tiene lugar.

A la luz, pues, de todo lo expuesto hasta el momento, parece posible extraer ahora una serie de conclusiones sobre los procesos de dialectalización que históricamente experimenta el léxico del español americano, en este caso ejemplificado por medio de la carta en la que García de Palacios describe el actual territorio salvadoreño.

En primer lugar, y como era de esperar, es necesario señalar que el análisis del corpus seleccionado ofrece un conjunto de vocablos que evidencia los procesos de adaptación que se están analizando, los cuales sirven para transformar el espanól en una herramienta de expresión útil y adecuada a la nueva realidad en la que se asienta; a este respecto, es especialmente destacable la rapidez con que se producen tales fenómenos, ya en la segunda mitad del siglo XVI, así como que aparezcan en el escrito de un peninsular emigrado, pues esta circunstancia no solo constata la vitalidad americana de los mismos - habida cuenta de que García de Palacio por fuerza ha de incorporar estas voces a raíz de sus vivencias en el Nuevo Mundo-, sino que además pone de manifiesto una vez más la aproximación del «idiolecto del hablante a la configuración léxica del dialecto dominante por medio de su parcial reestructuración" (Ramírez Luengo, 2013: 170) que se produce en las situaciones de contacto interdialectal.

Junto a esta primera constatación, el análisis desarrollado en estas páginas demuestra también que las estrategias con las que se van a llevar a cabo los procesos descritos van a ser fundamentalmente dos, la modificación semántica de voces endohispánicas y la incorporación de vocablos nuevos, generalmente tomados de las

americanismo puro (Company, 2010: XVII), en puridad un tipo extremo del anterior que reduce su frecuencia en el español europeo al mínimo posible. 
lenguas autóctonas americanas; por supuesto, el corpus ofrece ejemplos de ambas, pero es interesante resaltar que, a la luz de estos datos, García de Palacio parece mostrar una especial preferencia por la incorporación, que alcanza porcentajes de casi el $65 \%$ del total de los vocablos, si bien la configuración léxica puede presentar diferencias de peso en campos nocionales específicos, los cuales muestran diferencias porcentuales importantes que muy probablemente estén determinadas por distintas circunstancias de naturaleza sociohistórica.

Por último, es necesario mencionar la presencia en el texto de una tercera estrategia de americanización que tiene que ver con la generalización, en una variedad diatópica concreta, de vocablos presentes en el español general, que se transforman así en índice de dialectalización por mor de su frecuencia de uso; se trata del fenómeno cuantitativamente más escaso en el corpus, pero no por ello carece de interés, habida cuenta de que también contribuye, de manera sutil pero importante, a los procesos que van a aportar personalidad propia al vocabulario de las distintas variedades del español que son empleadas en el Nuevo Mundo.

Así pues, son varios los mecanismos que el español americano pone en marcha a la hora de responder a una situación claramente insatisfactoria como es la incapacidad de nombrar la realidad del nuevo continente; ante tal dificultad, las soluciones no tardan de aparecer, e implican una auténtica reestructuración del nivel léxico por medio de la modificación semántica de algunas voces endohispánicas, la selección y generalización de otras y la incorporación de nuevos vocablos tomados de las lenguas indígenas, algo que va a terminar por configurar la originalidad que presenta este nivel lingüístico en el Nuevo Mundo y a lo que ni siquiera los peninsulares emigrados, como bien demuestra la relación de García de Palacio, son capaces de sustraerse.

\section{APÉNDICE}

\section{Voces citadas en el trabajo}

ají, almácigo, anime, arfar, axín, bálsamo, cacao, cacique, caimán, calpul, chalchihuite, chián, ciruelo, copal, cote, cu, elote, encomendar, encomendero, estancia, hule, indio, invierno, jicara, jicol, maiz, maxtli, mazorca (de cacao), milpa, mitote, mojarra, nahuite, nata, piña, quetzal, sabana, tecuhtli, tehuamatini, teupa, teupixqui, venado, verano, xequipil, zapote, zontle. 


\section{BIBLIOGRAFÍA}

Álvarez de Miranda, Pedro (2009): «Neología y pérdida léxica», en E. de Miguel (ed.), Panorama de la lexicologia, Barcelona: Ariel, 133-58.

Arróniz, Othón (1994): El despertar científico de América. La vida de Diego García de Palacio, Ciudad de México: Universidad Autónoma Metropolitana.

Buesa Oliver, Tomás y José M. Enguita Utrilla (1992): Léxico del español de América: su elemento patrimonial e indígena, Madrid: MAPFRE.

Carriazo Ruiz, José Ramón (2002): Contribución a la historia del registro náutico y naval del Siglo de Oro, tesis doctoral inédita, Salamanca: Universidad de Salamanca.

Company, Concepción (2010): «Introducción», en Academia Mexicana de la Lengua, Diccionario de Mexicanismos, México DF: Siglo XXI, XV-XxıII.

Corde. Real Academia Española (2019): CORDE. Corpus diacrónico del español. URL: http://corpus.rae.es/cordenet.html; 28/07/2019.

Cuesta Domingo, Mariano (1994): "García de Palacio, escritor castrense y de náutica», Revista de historia naval 46: 79-98.

Damer. Asociación de Academias de la Lengua Española (2010): Diccionario de Americanismos, Madrid: Santillana.

DCECH. Corominas, Joan y José Antonio Pascual (1980-1991): Diccionario crítico etimológico castellano e hispánico, Madrid: Gredos.

Dle. Real Academia Española (2014): Diccionario de la lengua española, Madrid: Espasa-Calpe.

Escalante Arce, Pedro A. (2000): Cartas de relación y otros documentos. Pedro de Alvarado. Diego García de Palacio. Antonio de Ciudad Real, San Salvador: CONCULTURA.

Escalante Arce, Pedro A. (2019): «García de Palacio, Diego», en Real Academia de la Historia, Diccionario Biográfico electrónico. URL: http://dbe.rah.es/biografias/16273/diego-garciade-palacio; 01/07/2019.

Frago, Juan A. (1999): Historia del español de América. Textos y contextos, Madrid: Gredos.

García-Macho, M. Lourdes (2004): El léxico de la «Instrución náuthica para el buen uso y regimiento de las naos, su traça y govierno conforme a la altura de México" de Diego García de Palacio, Madrid: UNED.

Kero, Mats (2002): «El vocabulario náutico de Diego García del Palacio (1587): un análisis de términos de origen escandinavo antiguo", Revista de historia naval 78: 73-90.

Mejías, Hugo A. (1980): Préstamos de las lenguas indígenas en el español americano del siglo XVII, México DF: Universidad Nacional Autónoma de México.

Morínigo, Marcos A. (1998): Nuevo Diccionario de Americanismos e Indigenismos, Buenos Aires: Claridad.

Polo Cano, Nuria (2005): «Algunos indigenismos léxicos en el español de Guatemala del siglo XVIII», Res Diachronicae 4: 185-202. URL: https://resdi.net/volumen-iv/; 27/06/2019.

Ramírez Luengo, José Luis (2007): Breve historia del español de América, Madrid: ArcoLibros.

Ramírez Luengo, José Luis (2012): «El léxico en los procesos de dialectalización del español americano: el caso de la Bolivia andina», Cuadernos del Instituto de Historia de la Lengua Española 7: 393-404. 
Ramírez Luengo, José Luis (2013): «Notas sobre el contacto interdialectal en la historia de la lengua: un ejemplo del español del País Vasco en el siglo xvinı, Oihenart, 28. Actas de las IV Jornadas de Lingüistica Vascorrománica: 159-172.

Ramírez Luengo, José Luis (2014): "Contacto interdialectal en la historia del léxico: los americanismos en la Relación de Aller (Móxos, Bolivia; 1668)», en J.M. Santos Rovira (ed.), Fronteras y diálogos. El español y otras lenguas, Lugo: Axac, 165-176.

Ramírez Luengo, José Luis (20I7): «Aspectos metodológicos para el estudio histórico del léxico americano: conceptos, ejemplificación y tareas para el futuro», Moenia 23: 603-619.

Ramírez Luengo, José Luis (2019): «La Descripción Geográfico-Moral del arzobispo Cortés y Larraz (1770) y la historia léxica de Centroamérica: algunos datos salvadoreños», Cuadernos de Lingüistica de El Colegio de México 6/1: 1-30.

Ramírez Luengo, José Luis (en prensa): «Los indigenismos léxicos en la Descripción geográfico-moral del arzobispo Cortés y Larraz (1770): los datos salvadoreños», Revista de Lingüistica y Filología de la Universidad de Costa Rica 45/2.

Sainz López-Negrete, Miguel (1978): «Un montañés del siglo xvi: Diego García de Palacio», en Santander y el Nuevo Mundo, Santander: Centro de Estudios Montañeses, 407-429.

Santamaría, Francisco Javier (1959): Diccionario de mejicanismos, México DF: Porrúa. 
